\title{
Les interprètes, une clé vers l'égalité des chances
}

\author{
Carlos Quinto ${ }^{a}$, Robin Rieser ${ }^{\mathrm{b}}$, Barbara Weil ${ }^{\mathrm{C}}$ \\ ${ }^{a}$ Dr méd., membre du Comité central de la FMH, responsable du département Santé publique et professions de la santé; b collaborateur scientifique, \\ division Santé publique de la $\mathrm{FMH}_{;}{ }^{\mathrm{c}}$ cheffe de la division Santé publique de la FMH
}

\begin{abstract}
La communication entre le personnel médical et les patients est un élément crucial pour assurer le succès d'un traitement. Les différences linguistiques et culturelles peuvent entraver, voire rendre impossible le dialogue entre les personnes concernées.
\end{abstract}

Sur le plan mondial, la migration entraîne un mélange plus important des langues et des cultures, créant des difficultés de compréhension qui ne permettent souvent plus de garantir l'égalité des chances en matière d'accès aux soins. Dans le meilleur des cas, les interprètes communautaires professionnels peuvent aider à surmonter ces obstacles. Le présent article propose un tour d'horizon de la situation de l'interprétariat communautaire dans le domaine de la santé en Suisse.

\section{La diversité, un réel défi}

La Suisse est un pays de tradition plurilingue avec ses quatre langues nationales officielles. Les différentes cultures linguistiques se rencontrent, se mélangent ou se distancient. En ce sens, la «barrière de rösti» entre Suisse romande et Suisse alémanique est certainement l'exemple le plus parlant. En Suisse, quatre personnes sur dix parlent deux langues une fois par semaine, une sur cinq en parle trois et $7 \%$ en parlent quatre ou plus.

Près de $10 \%$ des personnes issues de la migration (première génération) indiquent ne maîtriser aucune des langues nationales.

Près d'un quart de la population n'est pas originaire de Suisse, les langues maternelles des étrangers vivant en Suisse s'ajoutent donc à nos langues nationales. Certaines personnes issues de la migration, et plus particulièrement celles avec un faible niveau de formation, ne maîtrisent qu'en partie ou pas du tout les langues nationales et sont donc désavantagées dans divers domaines. L'éducation, la santé et l'environnement social ne sont que quelques-uns des domaines concernés. Le manque de compréhension et les malentendus font qu'il leur est difficile de se faire comprendre et de s'impliquer en faveur de leur santé [1].

\section{L'accès aux soins est plus difficile pour les personnes défavorisées}

Les personnes avec un niveau de formation plus faible, un revenu plus bas ou une situation professionnelle plus difficile ont un risque plus élevé de contracter différentes maladies et leur espérance de vie est plus courte. Leur comportement en matière de santé est moins favorable, elles font moins de sport et mangent plus mal. Ce groupe de population est donc d'autant plus dépendant d'une prise en charge médicale de bonne qualité. En Suisse, les personnes migrantes ont souvent des difficultés à s'exprimer: près de $10 \%$ des personnes issues de la migration (première génération) indiquent ne maîtriser aucune des langues nationales. Afin de les aider à franchir ces barrières linguistiques, certains établissements de soins n'hésitent pas à engager des médecins titulaires de diplômes étrangers. Dans la plupart des cas cependant, médecin et patient doivent se comprendre sans soutien professionnel. Il arrive donc fréquemment que des parents, des connaissances ou les enfants des personnes concernées se chargent de la traduction lors de la consultation médicale. Bien que ces traducteurs non professionnels soient utiles dans la plupart des cas, cela n'est pas sans poser de problèmes: traductions incomplètes, rétention délibérée d'informations, évitement de questions taboues ou désagréables peuvent entraîner la réalisation d'examens et de traitements inutiles ou au contraire priver les patients des traitements nécessaires. Il en résulte des coûts inutiles et de la frustration de part et d'autre. La traduction par des 
interprètes professionnels tient compte des subtilités linguistiques des langues source et cible, des contextes culturels des deux parties, mène à une meilleure adhésion thérapeutique, améliore la qualité du traitement et la sécurité des patients [1].

\section{Quelle est la situation en Suisse?}

En Suisse, depuis plus de 15 ans, les professionnels de santé peuvent recourir à des interprètes professionnels pour les situations dans lesquelles la communication est difficile en raison de barrières linguistiques. En 2019, les interprètes communautaires sont intervenus plus de 163159 fois dans le domaine de la santé [2]. Une enquête réalisée en 2017 a montré que deux tiers de ces

\section{La FMH exige de clarifier les divergences en matière d'organisation et de rémunération du recours aux interprètes communautaires.}

interventions avaient lieu dans le domaine ambulatoire et plus de la moitié des généralistes et des pédiatres ont indiqué qu'une fois par mois au moins, il ne leur était pas possible de communiquer de manière directe et adéquate en consultation. De plus, ce problème est accentué par le fait que $44 \%$ des généralistes et pédiatres déclarent de pas savoir comment organiser l'intervention d'interprètes communautaires, 53\% indiquent ne pas vraiment savoir qui finance ces offres et $58 \%$ que le principal obstacle est l'organisation de ces interventions jugée plutôt fastidieuse. A cela s'ajoute le fait que leur financement varie d'un canton à l'autre et que seul un médecin sur dix sait comment ces prestations sont remboursées. Si les médecins en avaient connaissance, ils auraient huit fois plus souvent recours aux services d'interprètes communautaires. En règle générale, le remboursement de ces interventions qui, pour la plupart, ont lieu dans le domaine ambulatoire, n'est malheureusement pas garanti. Le TARMED ne prévoit pas la rémunération de telles prestations, mais celle-ci pourrait être négociée entre les partenaires tarifaires et introduite dans le TARDOC. Un autre obstacle de taille concerne les limites de temps dans le TARMED. Le Conseil fédéral est d'avis que la question du financement doit se régler entre partenaires tarifaires et ne voit aucune nécessité d'agir; une raison de plus pour que le Conseil fédéral approuve enfin le TARDOC [3].

\section{Que va-t-il se passer maintenant?}

Il n'est donc guère étonnant qu'au vu de la situation, deux motions $[4,5]$ relatives à l'interprétariat communautaire professionnel dans le domaine ambulatoire aient été déposées à Berne. La FMH exige quant à elle de clarifier les divergences en matière d'organisation et de rémunération du recours aux interprètes communautaires. Une réglementation uniforme valable pour toute la Suisse est indispensable pour garantir dans chaque région l'accès aux prestations d'interprètes communautaires afin d'assurer une prise en charge médicale équitable, avantageuse et de bonne qualité. A l'art. 41, al. 1bis, la Constitution fédérale précise: «La Confédération et les cantons s'engagent, en complément de la responsabilité individuelle et de l'initiative privée, à ce que toute personne bénéficie des soins nécessaires à sa santé.» [6] De plus, l’Office fédéral de la santé publique (OFSP) recommande de recourir aux services d'interprètes communautaires lors de conversations complexes et émotionnelles avec des patients allophones [7]. Pour toutes ces raisons, il est temps que la Confédération et les cantons agissent pour améliorer la prise en charge des patients allophones en Suisse et pour offrir le soutien nécessaire aux médecins et au personnel médical.

\section{Références}

1 Jaeger F. Les interprètes dans les cabinets suisses de médecine de famille et de pédiatrie. Etude mandatée par le Collège de médecine de premier recours (CMPR). Binningen; 2017. https://kollegium.ch/ sites/default/files/images/20170824_Dolmetschende_frz.pdf.

2 INTERPRET. Statistiques des interventions en interprétariat communautaire et médiation interculturelle pour l'année 2019;2020. www.inter-pret.ch/admin/data/files/marginal_asset/file fr/489/2019 einsatzstatistik fr.pdf?lm=1605533382.

3 Jaeger F, et al. Barriers to and Solutions for Addressing Insufficient Professional Interpreter Use in Primary Healthcare. BMC Health Services Research. 2019;19(1):753. https://doi.org/10.1186/s12913-0194628-6.

419.4279 > Nécessité de rembourser le recours à des interprètes dans les cabinets médicaux et en ambulatoire > Objet > Le Parlement suisse (parlament.ch).

$519.4357>$ Médecins et patients, comprendre et être compris. Financement des interprètes communautaires dans le domaine ambulatoire $>$ Objet $>$ Le Parlement suisse (parlament.ch).

6 RS 101-Constitution fédérale de la Confédération suisse du 18 avril 1999 (admin.ch).

7 www.bag.admin.ch/bag/fr/home/strategie-und-politik/nationale-gesundheitsstrategien/gesundheitliche-chancengleichheit/ interkulturelles-dolmetschen.html. 